\title{
A CONTRACULTURA DA DÉCADA DE 60 E A CENA INDIE DE JOGOS DIGITAIS
}

Cecilia da Fonte Alves

cecidafonte@gmail.com

Resumo: O presente artigo inicia uma discussão sobre as similaridades existentes entre a contracultura da déc. de 60 - fenômeno que, desde a sua origem, vem influenciando as práticas culturais independentes nas mais diversas áreas - e a atual cena indie de jogos digitais. Com tal objetivo, serão demonstradas duas características que são compartilhadas pelos manifestantes da contracultura e três desenvolvedoras da produção indie de games (Anna Anthropy, Merritt Kopas e Mattie Brice): seus comportamentos pessoalistas e comunitários. Acredita-se que relacionar tais profissionais a uma das manifestações que mais inspirou a cultura independente ao longo do tempo, pode ser uma maneira de gerar ferramentas para uma melhor compreensão dos valores que são praticados no cenário independente de jogos, assuntos que ainda são pouco explorados no meio acadêmico.

Palavras-chave: Independente, Indie, Jogos Digitais, Contracultura.

\begin{abstract}
This article begins a discussion on the similarities between the counterculture of the sixties - phenomenon that since its origin, has influenced the independent cultural practices in various areas - and the indie game scene. With that goal, this discussion will demonstrate two characteristics that are shared by the counterculture's participants and three developers of the indie games production (Anna Anthropy, Merritt Kopas and Mattie Brice): their appreciation for the personal and the community ethics. It is believed that relating such developers to one of the manifestations that most inspired independent culture over time, can be a way to generate tools for a better understanding of the values that are practiced in the independent game scene, issues that are still little explored in academic papers.
\end{abstract}

Keywords: Independent, Indie, Digital games, Counterculture. 


\section{INTRODUÇÃO}

O setor independente dos jogos digitais tem crescido exponencialmente na última década, tornando-se cada vez mais amplo e heterogêneo. O surgimento de métodos de distribuição online, ferramentas gratuitas de desenvolvimento e crowdfunding é um dos fatores que possibilitaram o aumento da produção e da popularidade dos games indies, que no geral são produzidos por uma pessoa ou pequenas equipes e sem o apoio ou suporte financeiro de empresas de publicação. Comumente experimentais e envoltos por uma vasta liberdade criativa, muitos desses jogos fogem das características do setor mainstream ${ }^{1}$, sendo criados a partir de um desejo de seus desenvolvedores de democratizarem a produção, distribuição e circulação de games, ao mesmo tempo em que fornecem perspectivas de resistência à crescente aproximação da grande indústria, que vê nos títulos independentes um enorme potencial financeiro e criativo.

Este artigo busca, então, estudar esta cena independente de jogos que procura desafiar o ambiente corporativo, observando-a em um contexto mais amplo, a partir das semelhanças que a mesma compartilha com o movimento de contracultura da déc. de 60 - fenômeno que deu origem a inúmeros grupos radicais e influenciou de maneira bastante representativa toda a cultura posteriormente chamada de indie. Com esta finalidade, serão demonstradas inicialmente duas características que são compartilhadas pelos manifestantes da contracultura e três representantes dessa produção indie de games (Anna Anthropy, Merritt Kopas e Mattie Brice): seus comportamentos pessoalistas e comunitários. Mencione-se que ambos os cenários serão analisados no contexto dos Estados Unidos.

Dada a importância crescente do setor independente dos games, que ainda é pouco explorado no meio acadêmico, acredita-se que aproximar tais desenvolvedoras a uma das manifestações que mais inspirou as práticas independentes no decorrer da história, pode ser uma maneira de gerar ferramentas para um melhor entendimento dos valores que são praticados no cenário indie de jogos e, até, de ver essas profissionais como perpetuadoras dos ideais de uma cultura independente mais ampla. Espera-se, ainda, que a presente pesquisa traga reflexões acerca das diferenças que existem dentro do próprio universo de jogos chamado de indie, assim como da utilização de artefatos de Design como ferramentas de expressão pessoal e política.

Além da introdução, a composição deste documento envolve três seções. A primeira incluirá um estudo da contracultura da década de 60, o qual buscará discutir sobre como os participantes do movimento se relacionavam através de comportamentos extremamente pessoalistas e comunitários e se organizavam com o intuito de propagar seus ideais e galgar espaços de criações mais livres. Tal discussão trará como principal referência o escritor e historiador Theodore Roszak, que vivenciou o fenômeno da contracultura. Na segunda seção será feita uma descrição da cena independente dos games, quando será possível perceber o quanto a mesma tem se tornado diversa, e como alguns de seus criadores, similarmente aos participantes da

\footnotetext{
${ }^{1} \mathrm{O}$ termo mainstream, que se traduz para o português como "principal corrente", está associado à cultura dominante e à produção de massa. Refere-se a um meio ou um conteúdo que popular, que tem apelo comercial e está inserido na lógica capitalista. Os jogos mainstream, conforme Ferreira (2014, p.4), geralmente são aqueles desenvolvidos e distribuídos (publicados) por grandes empresas (ex: Microsoft, Sony, Nitendo, etc.) e que envolvem "centenas ou às vezes milhares de funcionários e grandes cifras de capital".
} 
contracultura - enquanto buscam transformar muitas das práticas da cultura dominante de jogos - valorizam as trocas pessoais e coletivas. Nesse momento serão destacados vários depoimentos das desenvolvedoras independentes estudadas, com o intuito de demonstrar a real essência de suas criações e pensamentos. Na última seção serão levantadas as conclusões desta pesquisa, assim como suas limitações e possíveis desdobramentos.

\section{A CONTRACULTURA DA DÉC. DE 60 E O SEU CARÁTER PESSOAL E COMUNITÁRIO}

Roszak (1972, p.19), que popularizou o termo "contracultura" em 1969, explica que esse fenômeno surge como uma contestação da tecnocracia, que entre as décadas de 60 e 70, era mais desenvolvida nos Estados Unidos do que em qualquer outra sociedade. Para o autor, a tecnocracia:

É aquela forma social na qual uma sociedade industrial atinge o ápice de sua integração organizacional. É o ideal que geralmente as pessoas têm em mente quando falam de modernização, atualização, racionalização, planejamento (ROSZAK, 1972, p.19).

Nesse sentido, entende-se que a razão científica e a objetividade tornavam-se dominantes na nação americana que vivenciava a Guerra Fria, período de disputa hegemônica com a União Soviética, no qual o progresso tecnológico e a corrida armamentista nuclear refletiam-se no funcionamento de toda a sociedade. A contracultura surge, então, como um movimento de recusa ao status quo, sendo criada, principalmente, por uma juventude que buscava valores mais humanos e pessoalistas, no lugar de princípios técnicos, industriais e de racionalidade. Paes (1997, p. 22) comenta esse fenômeno:

(...) Para milhões de jovens naquela década, a saída vislumbrada foi a busca de um mundo alternativo. Da recusa da cultura dominante e da crítica ao "sistema" (como então se dizia), nasceram novos significados: um novo modo de pensar, de encarar o mundo, de se relacionar com as outras pessoas (PAES, 1997, p. 22).

O desejo de mudar o mundo e a luta contra as desumanidades da tecnocracia fizeram surgir manifestações diversas como o movimento hippie e a Nova Esquerda estudantil, por exemplo, e a valorização das religiões orientais, o culto à natureza; ao comunitarismo; a luta pela paz; respeito às minorias raciais e culturais; entre outros princípios (SOUSA, 2013). E no contexto da cultura independente, inúmeros grupos radicais emergiram, buscando estilos de vida e trabalhos mais livres e servindo como um guia para diversas atividades culturais subsequentes. Kaya Oakes, em seu estudo sobre a cultura independente norte-americana, argumenta, por exemplo, que foram os artistas independentes dos anos 50 e 60 das gerações beat e hippie que - ao trabalharem de maneira distinta da indústria mainstream, valorizando a criatividade acima do lucro - fundamentaram toda a cultura, mais tardiamente, nomeada de indie. (OAKES, 2009, grifo da autora, p. xii-31 passim).

Ao explicar as insatisfações da juventude dos anos $60 \mathrm{com}$ a predominante racionalidade científica da tecnocracia - que teriam motivado a geração da contracultura - Theodore Roszak (1972, p. 68), destaca a alienação para com as relações humanas. Isto ocorre, segundo o autor, numa sociedade que se baseia na 
objetividade, na coisificação de tudo e de todos, ou seja, não há espaço para a subjetividade e envolvimento pessoal:

Quando pedimos a uma pessoa que seja objetiva queremos dizer que ela deve evitar falar sobre seus sentimentos interiores e que deve olhar o mundo como um engenheiro olha um projeto de construção (ROSZAK, 1972. p. 64).

Sob o domínio da tecnocracia e dos seus especialistas, o homem perde então sua sensibilidade, sua intuição, sua confiança em si mesmo, vendo-se dependente e à parte do conhecimento:

Num mundo mecânico e despersonalizado, o homem tem uma sensação indefinível de perda; uma sensação de que a vida se tornou empobrecida (TAYLOR, Apud JOSEPHSON e JOSEPHSON, 1962, p. 11).

Diante dessa organização social racional e objetiva, as manifestações contraculturais dos anos 60 surgem como uma oposição ao sentimento de alienação, buscando reativar as sensibilidades humanas, as experiências pessoais e as subjetividades renegadas pelo sistema industrial. O fenômeno, que é conduzido principalmente por pessoas da classe média, se volta aos valores pessoalistas e comunitários, aos sensos naturais e instintivos das tribos e aldeias primitivas.

Dentre os principais mentores e influenciadores do movimento, é cabível destacar Frank O'hara que contribuiu de maneira significativa para a formação dos valores da contracultura e das suas práticas independentes. Nascido em 1926 nos Estados Unidos, O'hara, junto a outros artistas e poetas, pertenceu ao grupo intitulado "A Escola de Nova York", cuja popularidade data dos anos 50. Segundo Oakes (2009, p. 21, 22) o grupo buscava quebrar tabus e fazer experimentações em seus trabalhos criativos e - inserido em uma cena na qual ser artista não mais significava ter tido um treinamento específico ou um patrocinador - fazia a arte que queria e porque queria: "o que desse nos nervos" (frase de Frank O'hara). Deste modo, os participantes da Escola de Nova York não consideravam a poesia como algo reservado para a academia e sim como parte de sua experiência diária. Oakes (2009, p. 22) explica que os assuntos abordados por O'hara em seus escritos envolviam temas banais como passeios, comidas, encontros com amigos, etc. Seguindo o lema do escrever sobre o que desse nos nervos, os artistas independentes daquele tempo formaram a base para a cultura indie de hoje. Outra característica da Escola de Nova York que inspirou a geração contracultural dos anos 60 diz respeito à valorização das relações pessoais e do respeito à diversidade. Segundo Oakes (2009, p.22), O'hara, que era homossexual, tinha uma grande estima pela amizade e atraía para perto de si pessoas de raças e orientações sexuais diversas.

A personalidade extremamente pessoalista de O'hara também se refletia no seu instinto comunitário. Oakes (2009, p. 24, 25) argumenta que ele promoveu a ideia de que a organização comunitária é fundamental para o trabalho criativo e que em sessões de leitura, não apenas lia seus poemas mas também os daquelas pessoas que faziam parte do seu círculo de amizades. Cabe destacar que os livros de poemas de Frank O'hara, que para muitos era um vanguardista, eram distribuídos muitas vezes através de sua rede de amigos ou através de editoras independentes como a City Lights que em 1964 lançou o título "Lunch poems" do autor. 
Frank O'hara também é associado aos beats, cujos ideais influenciaram enormemente os hippies dos anos 60 . Remontando às décadas de 40 e 50 , a geração beat era formada por escritores e escritoras - entre eles Allen Ginsberg e Diane Di Prima - que através de suas publicações, rejeitavam os padrões da cultura mainstream, abordando temas como sexo, drogas e amores não-convencionais (JESSMER, 2012, p.4). Ao explorarem assuntos que nada representavam as visões da literatura dominante, segundo Spencer (2008, p. 84, 85) os beats tiveram seus trabalhos rejeitados pela grande imprensa e desta forma decidiram criar seus próprios meios de produzir e distribuir textos, se utilizando de métodos mais autônomos de publicação. A autora ainda observa que a publicação independente dos beats se baseava na cooperação, quando podemos verificar o senso comunitário tão valorizado pela contracultura dos anos 60: "Esses escritores dos anos 40 e 50 geralmente se apoiavam e ajudavam a publicar os trabalhos uns dos outros". (SPENCER, 2008, p. 86).

Paul Goodman também foi um dos grandes mentores da contracultura e seu "espírito independente feroz, compromisso com os valores democráticos e simpatia com as preocupações dos jovens " fizeram dele um dos mais importantes modelos para a Nova Esquerda (SILVERMAN, 2011). A obra vasta de Goodman envolvia poesias, sócio-novelas, críticas literárias e sociais. Mas, apesar de toda a sua intelectualidade, Goodman se opunha aos padrões do academicismo pois, em seu discursos, surgia como um homem acessível expondo suas ideias e princípios a partir do que era e não apenas pelo que sabia:

Enquanto a atitude acadêmica habitual é pomposa, remota, defensivamente disfarçada numa estreita especialização, Goodman surge como um homem inteiro e vulnerável. Como se dissesse "a verdade é tanto uma questão do que sou quanto do que eu sei. Por isso vou mostrar-lhes o que eu sou" (ROSZAK, 1972, p.196).

Roszak (1972, p.196) explica que esse desarmamento psíquico de Goodman demonstrava sua necessidade de expor seus sentimentos, o que se assemelhava aos escritores beats e aos hippies. Entretanto, ainda de acordo com o autor, a maior contribuição de Goodman à contracultura foi o seu comunitarismo: "Só uma sociedade que possua a elasticidade de comunidades decentralizadas é que pode absorver as inevitáveis falibilidades do homem" (ROSZAK, 1972, p. 204). Os pensamentos anarquistas e voltados à democracia participante de Goodman fizeram dele um teórico influente da Nova Esquerda, assim como também inspiraram diversos grupos como os hippies, as comunidades psicodélicas e os diggers dos anos 60.

Examinando alguns dos principais movimentos contraculturais da década de 60, como a Nova Esquerda estudantil e os hippies, percebe-se que esses grupos eram bastante divergentes. Roszak (1972, p. 66) pontua que, de um lado, estava a boemia hippie e, de outro, o ativismo político dos estudantes da Nova Esquerda. Entretanto, como se observou, ambos sofreram influências similares e, além de compartilharem o posicionamento de oposição à cultura dominante, continham ideais comunitários e eram fortemente pessoalistas.

Atuando entre estudantes e povos oprimidos, como negros e vítimas do imperialismo norte-americano, a Nova Esquerda "liderou movimentos contra a guerra do Vietnã, pelos direitos estudantis nas universidades e por maior liberdade na vida cotidiana" (PURDY, 2008). O grupo também valorizava a democracia participativa e se 
opunha à despersonalização da sociedade tecnocrática. Analisando particularmente os valores de um dos grupos da Nova esquerda, os Estudantes por uma Sociedade Democrática, Roszak (1972, p. 70) explica que esses ativistas priorizavam relações diretas e abertas com outras pessoas, ou seja, sua política era feita através de envolvimentos pessoais. "Opomo-nos à despersonalização que reduz os seres humanos à condição de coisas", afirmavam em uma declaração de 1962. (ROSZAK, 1972, p. 69).

Os hippies também valorizavam o envolvimento pessoal e os sentimentos interiores e praticavam a autoanálise, rumo a uma "viagem" ao interior. A postura pessoalista desse grupo também se ampliava para o exterior, para o coletivo, quando as relações humanas passavam a ser cooperativas, e o compartilhamento das informações horizontal e democrático. Esses "jovens tribalizados" procuravam inspiração nas relações humanas profundamente pessoais das aldeias e tribos, e segundo Roszak (1972, p. 154, 155), em seus rituais todos tinham acesso igual ao conhecimento. Nesse contexto, o autor comenta sobre a figura inspiradora do xamã tribal: "A essência da boa magia tal como praticada pelo xamã está em que ela procura sempre tornar acessível a todos o pleno vigor da experiência do mágico. (...) Seu dom peculiar confere responsabilidade e não privilégio" (ROSZAK, 1972. p. 260, 261).

As comunidades e fazendas cooperativas formadas pelos hippies e outros ativistas nos anos 60 também se baseavam, de maneira ainda mais potencial, em ideais pessoalistas e na democracia participante. Essas organizações se fundamentavam na ética faça-você-mesmo, ou seja, buscavam a auto-suficiência e a independência dos grandes sistemas capitalistas, procurando não depender de auxílios profissionais (WEHR, 2012, p. 25). Dentre tantas comunidades e fazendas cooperativas fundadas nos anos 60, podemos citar a "Black Bear Ranch" criada pelos Diggers ativistas e atores de improviso que organizavam concertos musicais e peças de teatro de rua, acreditando na importância da utilização livre dos espaços urbanos para as interações interpessoais. A busca pela independência dos Diggers também se refletia em sua fundação intitulada "The communication Company" através da qual publicavam e distribuíam livros e notícias, ações que podiam ser vistas em inúmeros outros impressos de uma grande imprensa underground que surgiu nos anos 60.

Conclui-se, nesta seção, que os manifestantes da contracultura da déc. de 60 estimavam as relações humanas profundamente pessoais e coletivas, estimulando, entre outras práticas, o acesso livre ao conhecimento e a troca de experiências. A seguir veremos como tais características se manifestam nos comportamentos de Anna Anthropy, Merritt Kopas e Mattie Brice - três game designers da cena indie de jogos.

\section{PESSOALISMO E COMUNITARISMO NA CENA INDIE DOS JOGOS DIGITAIS}

Nos últimos anos, o cenário independente de games se expandiu, ganhando cada vez mais o reconhecimento do público e do mercado. O surgimento e a democratização de ferramentas de desenvolvimento gratuitas e dos canais de distribuição online e de crowdfunding, por exemplo, são alguns dos fatores que facilitaram a criação e publicação de games de maneira mais autônoma. Mas o que são jogos independentes ou indies? Se pensarmos no sentido mais básico dos termos iremos considerar esses tipos de produções como aquelas que foram realizadas e distribuídas com bens próprios, sem contar com o suporte financeiro de uma empresa publicadora. Entretanto, as classificações de títulos independentes abrangem muito 
mais do que fatores econômicos e apresentam, frequentemente, interpretações divergentes.

Alguns argumentam, por exemplo, que as produções independentes são opostas àquelas criadas no ambiente corporativo. Nesse sentido, entende-se que games indies são desenvolvidos por pequenas equipes que ao realizarem atividades sem restrições contratuais e criativas, produzem jogos autorais e experimentais. Outros ainda consideram que criadores independentes são aqueles que têm uma atitude de oposição ao modelo de produção e distribuição da indústria comercial e aos valores que ela representa.

Por outro lado, há quem desconsidere, em suas qualificações de jogos independentes, a autonomia financeira do criador, informando que ele pode contar com o apoio de uma empresa publicadora, por exemplo, desde que não perca sua liberdade criativa, como opina o autor Graebesh: "A independência na produção não deve ser em direitos autorais mas em criatividade" (GRAEBESH, 2012, p.50).

A ausência ou não de um suporte externo, talvez seja um dos fatores que mais problematiza as classificações dos jogos independentes. Isto se demonstra, por exemplo, ao observarmos a gradual aproximação da indústria mainstream que, ao ver o potencial financeiro da produção independente nos últimos anos, ofereceu inúmeras oportunidades de publicação para muitos desenvolvedores indies, além de contratos para futuras produções ${ }^{2}$. Jogos como Super Meat Boy, Flower e Braid que geraram altíssimas receitas e foram produzidos de maneira autônoma, mas publicados através das plataformas de console da Sony e da Microsoft, para muitos são títulos independentes, enquanto que para outros, eles dificilmente podem ser intitulados como tal (DE JONG, 2013, p.16).

Diante de toda a problemática que cerca a conceituação de independência na área de jogos, e que, acredita-se, apresenta o cenário independente de games de maneira extremamente ampla e heterogênea, este artigo adota a visão de Paolo Pedercini que enxerga o termo indie como um gradiente infinito, no qual há inúmeros graus de dependência em relação ao capital. O autor argumenta que não existe independência absoluta, já que o modelo de produção da atualidade apresenta dependências diversas como aquelas tecnológicas e infra-estruturais. Isto é, mesmo aqueles desenvolvedores que estão mais distantes do ambiente corporativo ainda fazem uso de inúmeras ferramentas disponibilizadas pelo grande setor industrial. Deste modo, ele propõe que a independência seja enxergada como uma utopia:

(A utopia) impulsiona você a romper continuamente com as forças e as entidades que nos fazem infelizes e acabam com o mundo. (...) E é assim que eu gosto de pensar sobre a independência em jogos e na cultura. Não é um status, mas uma direção a seguir. O que devemos fazer é conspirar sobre como podemos ser mais autônomos. Sobre como podemos avançar mais um passo em direção à independência. (PEDERCINI, 2012).

\footnotetext{
${ }^{2}$ No contexto de estreitamento entre o ambiente corporativo e a produção indie, muitos chegaram a proclamar o fim da cena independente - principalmente entre os anos 2013-2015. Disponível na internet por http: <http://www.ign.com/articles/2014/07/29/why-do-so-many-people-hate-indiegames>, <http://artery.w bur.org/2014/01/28/indie-games-died>, <http://www.n ewstatesman.com/culture/2014/07/why-indie-gaming-s-obsession-moneymaking-hurts-us-all>, <http://www.pocketgamer.biz/stateside/61563/theind ie-dream-is-dead/>. Acesso em 18 abr. 2016
} 
Ao considerarmos a independência como algo a ser almejado, nos atentaremos neste artigo para um nicho de desenvolvedores de jogos que, em meio a esse imenso e plural universo indie, buscam formas mais autônomas de produzirem e distribuírem seus games, além de possuírem posturas políticas que estimulam a democratização social na área de jogos ${ }^{3}$. Anna Anthropy, Merritt Kopas e Mattie Brice são três desenvolvedoras de games que possuem tais motivações, e que se fundamentam no pessoalismo e nos valores comunitários, enquanto demonstram insatisfações com a indústria mainstream de jogos e até mesmo com a cena indie que se aliou ao ambiente corporativo.

Ao comparar os grandes publicadores de jogos à produção independente que gerou um sucesso enorme comercial, Anna Anthropy afirmou não ver diferenças entre as mesmas: "eles são basicamente a mesma coisa: um monte de homens brancos fazendo dinheiro ao refazer os mesmos jogos continuamente. (ANTHOROPY, 2013).

A desenvolvedora, que acredita que a grande indústria de games apresenta diversas barreiras para o acesso de muitos, discute com frequência não apenas sobre a necessidade de um universo que agregue uma pluralidade de gênero, raças, classes sociais, mas a inclusão daqueles que tradicionalmente não fazem parte dessa cultura, que segundo ela "tende a ser dominada apenas por perspectivas muito privilegiadas" (ANTHROPY, 2016).

Mattie Brice, desenvolvedora e crítica de games negra e transgênero, também não demonstra, através dos textos que escreve, compartilhar das mesmas motivações daqueles que se intitulam de indies, assim como Merritt Kopas, que relembrando o início de sua experiência como desenvolvedora de jogos, informa que não se sentia representada até mesmo pelos desenvolvedores independentes. (KOPAS, 2015, p. 6).

A rejeição ao que o cenário mainstream de videogames e a produção indie "high profile" representam, e o desejo de tornar o universo de jogos mais inclusivo das três desenvolvedoras aqui analisadas, se refletem na forma como criam e distribuem seus jogos assim como na maneira que se relacionam entre si e com o público.

Verifica-se que elas escolhem ferramentas de desenvolvimento de jogos de baixo custo ou gratuitas como Twine, Unity e Game maker ou, como pontua Mattie Brice em seu site pessoal: "plataformas acessíveis nas quais pessoas sem conhecimento de programação ou verbas podem desenvolver games". Também é comum que disponibilizem suas produções para serem jogadas online ou que o download possa ser feito de maneira gratuita através dos seus sites pessoais ou em canais de distribuição online como o Dropbox e o itch.io. Os títulos são comumente gratuitos e se cobrados, seus preços variam entre $\$ 1$ e $\$ 5$.

Dentre essas ferramentas utilizadas por Mattie, Anna e Merritt, cabe destacar a Twine, software open-source criado em 2009, mas que ganhou uma enorme popularidade no ano de 2014. A plataforma, que não exige experiência prévia em programação, para Merritt Kopas (2015), oferece à área de games um ambiente mais plural e democrático:

Utilizada por autores de jogos não-tradicionais para descreverem assuntos distintamente não-tradicionais, o movimento Twine nos dias de hoje tem oferecido um espaço para aqueles que previamente não possuíam voz na

\footnotetext{
${ }^{3}$ Destaca-se que elas produzem jogos para computador e não para consoles, já que procuram meios de publicação alternativos.
} 
cultura de jogos para contarem suas próprias histórias e inventarem novas visões fora dos canais tradicionais do mercado (KOPAS, 2015).

O software, que serve como ferramenta de criação de histórias não-lineares, possui um enorme potencial para expressões individuais, potencial este que motivou Merrit Kopas a dar o primeiro passo na criação de games em 2012. Ela explica que foi depois de jogar o game Twine "Encyclopedia fuckme and the case of the vanishing entree" (2011) de Anna Anthropy, que pela primeira vez sentiu a voz pessoal do criador em um jogo. Uma voz com a qual se identificou (KOPAS, 2015, p.6,7).

O contato com uma cena de desenvolvedores que narravam suas experiências pessoais, fez com que Kopas, que é transexual, se sentisse encorajada a usar videogames para se expressar. Hoje, com mais de 26 games produzidos, ela procura, através de suas produções, desafiar os preconceitos e as experiências dos jogadores focando, por vezes, em sua própria vida: "Eu quero fazer parte de uma comunidade e me conectar com pessoas que compartilham das minhas experiências. Me colocar através do meu trabalho tem sido uma boa maneira de fazer isso" (KOPAS, 2014). Em seu jogo Lim, lançado em 2012, o jogador controla um quadrado que navega em um labirinto habitado por quadrados violentos que tentam bloquear a passagem do mesmo evitando que ele prossiga. Entretanto, o quadrado principal tem a capacidade de se camuflar como se fosse igual aos outros, o que pode evitar bloqueios mas também pode gerar turbulências em si próprio. Em uma entrevista, Kopas procurou explicar quais eram os seus objetivos ao produzir Lim:

O que eu estava tentando fazer era criar um game que entrasse nos
diferentes tipos de violência que acontecem no não-espetacular cotidiano e
que geralmente não são explorados na área de jogos, e claro, muito disso
veio da minha experiência como transexual. (...) Passear em um espaço se
perguntando sobre como as pessoas verão você e quais são as
consequências disso (KOPAS, 2013).

Verifica-se que os games de Anna Anthropy e Merritt Kopas assim como os de Merritt também retratam suas experiências pessoais, ao mesmo tempo em que refletem seu engajamento com questões sociais e políticas ou como afirma Kareem (2015) em seu artigo "Os Jogos que são alternativos demais para a categoria indie": "À medida que esse diverso grupo de desenvolvedores mergulha no oceano do código aberto e dos softwares gratuitos, suas próprias experiências inspiram seus produtos".

Anna Anthropy, como já foi possível observar, rejeita a cultura dominante de games em vários sentidos, e um outro fator de insatisfação diz respeito à impessoalidade dos games desenvolvidos pelas grandes empresas: "Jogos mainstream são realmente impessoais" (ANTHROPY, 2013). Em seu livro "Rise of the videogame zinesters" lançado em 2012 ela propõe que a produção de jogos seja mais pessoal e intimista e que aborde temáticas tão simples quanto: "Seus desejos, seus sonhos, seus medos"(ANTHROPY, 2012, p. 138). Na publicação ela também argumenta que o videogame não é um produto, mas "uma criação de uma autora e sua testemunha, a jogadora. Feito pelas mãos da primeira e distribuído de maneira pessoal para a segunda" (ANTHROPY, 2012, p. 141).

Entre os jogos criados por Anthropy - que já desenvolveu inúmeros como Mighty Jill Off e Calamity Annie - Dys4ia foi um dos que mais trouxe reconhecimento para a desenvolvedora. O game, de cunho autobiográfico, explora a terapia de 
reposição hormonal vivida pela autora, que assim como Merritt e Mattie é transexual. Dys4ia faz com que o jogador vivencie não apenas as mudanças no corpo da personagem mas o contexto social que envolve a narrativa. "Todos os meus jogos são pessoais; todos eles são um reflexo de mim", afirmou Anna (ANTHROPY, 2012).

Similarmente, o game Mainichi desenvolvido por Mattie Brice se inspirou em sua própria vida como transexual enquanto buscou trazer reflexões sobre a marginalização de determinados grupos no universo de videogames:

Este é um experimento de partilha de uma experiência pessoal através de um sistema de jogo. Do mesmo modo, o jogo atua como um comentário a respeito de como utilizamos o game design para ampliar o acesso a experiências universais no lugar de hiper-pessoais, e como frequentemente excluímos vozes minoritárias (BRICE, 2012, grifo da autora).

Buscando encorajar a participação de mais pessoas na área de jogos, as desenvolvedoras aqui analisadas se envolvem em causas mais amplas e em atividades paralelas à criação de games, compartilhando conhecimento em seus sites, ao fornecerem tutoriais ou organizarem eventos. Além da publicação de livros e de textos diversos, Merritt Kopas realiza workshops, palestras e curadorias. Em seu site "Forest Ambassador", no qual apresenta diversos jogos e traz informações sobre os mesmos, a desenvolvedora procura se comunicar com aqueles que se sentem excluídos, intimidados ou mesmo não têm interesse por games (KOPAS). Similarmente, Anna Anthropy, além de desenvolver jogos, participa de palestras, ministra workshops e escreve textos com o intuito de divulgar processos de produção e distribuição que seriam mais acessíveis, como o zine intitulado "Make your first video game in twine". Mattie Brice escreve artigos que tratam de temáticas que são pouco ou nada abordadas pela grande mídia e também conduz eventos voltados para a discussão de design inclusivo e oferece consultorias.

Cabe mencionar que, sem o suporte de empresas publicadoras, essas profissionais utilizam sites de financiamentos coletivos como o Patreon ou disponibilizam em seus sites pessoais um espaço para doações, além de divulgarem seus trabalhos em suas páginas pessoais, blogs e contas de twitter. Além disto, verifica-se que, por possuírem ideais comuns, elas acabaram se aproximando, interagindo entre si e promovendo os games umas das outras, fatores que possibilitam uma maior visibilidade aos seus trabalhos. Merritt Kopas, que também organiza workshops com Anna Anthropy, em sua publicação "Videogames for humans" retratou as produções da mesma, por exemplo. No site de Mattie Brice, que similarmente já participou de eventos junto com as demais desenvolvedoras, há uma seção "Apoie críticos de games", na qual ela apresenta uma lista de autores que se dedicam ao campo de jogos, entre eles Merrit Kopas. Destaca-se ainda que elas contam com o auxílio dos jogadores e fãs que além de fazerem doações, participam de fóruns e constantemente dividem suas experiências com as mesmas, oferecendo um feedback que alimenta essa grande rede comunitária, da qual todos fazem parte.

\section{CONCLUSÃO}

Este artigo inicia um estudo da atual cena indie dos jogos digitais, buscando observá-la a partir de suas semelhanças com a contracultura da década de 60 fenômeno que serviu como fonte de inspiração para inúmeras práticas culturais 
independentes ao longo da história. Com este fim, foram demonstradas duas similaridades compartilhadas pelos participantes da contracultura e três desenvolvedoras independentes de games (Anna Anthropy, Merritt Kopas e Mattie Brice): a ética pessoalista e comunitária valorizada pelos mesmos.

Através das análises percebe-se, primeiramente, que os manifestantes da contracultura assim como as três profissionais estudadas, contestam, cada um à sua maneira, os valores estabelecidos pela grande indústria - seja por achá-la impessoal ou marginalizante, por exemplo. Observa-se que as desenvolvedoras de jogos são movidas por um desejo de democratizar a produção, distribuição e circulação de seus trabalhos, assumindo posturas políticas que visam a diversidade e o Design inclusivo e buscando ferramentas alternativas para realizarem e publicarem seus games, de maneira semelhante aos criadores da contracultura.

Demonstra-se ainda que, com tais motivações, tanto os hippies, estudantes da nova esquerda e outros indivíduos analisados no contexto da contracultura, e as três game designers estimam o pessoal e o comunitário, se fundamentando nas relações humanas extremamente intimistas e nas ações coletivas e cooperativas. Isto se apresenta na forma como se relacionam entre si e com o público, seja ao expressarem os seus sentimentos internos, colocando suas experiências de vida através de seus trabalhos, ou ao priorizarem o compartilhamento de conhecimentos e promoverem os trabalhos uns dos outros, como em uma grande rede comunitária.

Conclui-se, portanto, que é possível apontar algumas similaridades entre o atual cenário independente dos jogos digitais e a contracultura da década de 60 que para muitos é o berço dos criadores independentes. Entretanto, deve-se mencionar que a discussão aqui presente não está concluída, pois pela complexidade e heterogeneidade das duas temáticas não foi possível abordá-las com maior profundidade, cabendo dar continuidade a tais estudos futuramente.

Espera-se que este trabalho ofereça ferramentas para uma melhor compreensão da produção indie de jogos, que ganha notoriedade apenas nos últimos anos. Também acredita-se que os assuntos aqui analisados possam fornecer um embasamento para a origem de outras abordagens, e para fazer o leitor ou leitora refletirem sobre a heterogeneidade que envolve o universo indie de jogos ou os ideais que cercam as produções culturais chamadas de independentes e até a respeito da utilização de artefatos do Design como ferramentas de expressão pessoal ou como contestadores da própria indústria.

\section{REFERÊNCIAS}

ANTHROPY, Anna. Rise of the videogame zinesters. Nova York: Seven Stories, 2012.

Anna anthropy: games should share an idea, not rob us of time and money.

2012. Disponível na internet por http em: <http://www.gamesforchange.org/2012/03/ anna-anthropy-interview/>. Acesso em 20 abr. 2016

Road to the IGF: Anna Anthropy's Dys4ia. 2013. Disponível na internet por http em:<http://www.gamasutra.com/view/news/188242/Road_to_the_IGF_Anna_An thropys_Dys4ia.php>. Acesso em 8 maio. 2016

Anna Anthropy and the Twine revolution. 2013. Disponível na internet por http em: <http://www.theguardian.com/technology/gamesblog/2013/apr/10/annaanthropy-twine-revolution>. Acesso em 5 maio. 2016 
Ohmygod Are You Going To Be Alright? an interview on passion, perseverance and hope for the future with Anna Anthropy. 2016. Disponível na internet por http em: <https://modelviewculture.com/pieces/ohmygod-are-you-goingto-be-alright-an-interview-on-passion-perseverance-and-hope-for-the-future-withanna-anthropy/>. Acesso em 20 maio. 2016

BRICE, Mattie. Mainichi. 2012. Disponível na internet por http em: < http://www.mattiebrice.com/mainichi/>. Acesso em 10 maio. 2016

DE JONG, Joey. Indie Issues: The Meaning of 'Indie' Games, and Their Incorporation into the 'Mainstream' Game Industry. 2013. 42 f. Dissertação (mestrado) - Universiteit van Amsterdam, Curso de Pós-Graduação em New Media e Digital Culture.

FERREIRA, Emmanoel. Diz-me com quem andas e direi o quão 'indie' és: relações entre gênero e mercado no cenário dos videogames independentes. In: ESCOLA SUPERIOR DE PROPAGANDA E MARKETING. 2014, São Paulo. Anais do Congresso Internacional em Comunicação e Consumo, 4. São Paulo: ESPM, 2014. P. 1-15.

GRAEBESCH, Roman. The indie game. 2012. 100 f. Dissertação (mestrado) - University of Copenhagen, Curso de Pós-Graduação em Media Technology \& Games.

JESSMER, Anna Lou. Containing the Beat: An Analysis of the Press Coverage of the Beat Generation During the 1950s. 2012. 65 f. Dissertação (mestrado) - Marshall University, Curso de Pós-Graduação em Arts in Journalism.

JOSEPHSON, Eric; JOSEPHSON Mary. Man Alone: Alienation In Modern Society. Nova York: Eric and Mary Josephson, 1962.

KAREEM, Soha. The Games that are too underground to be indie. Disponivel na internet por http em: <http://motherboard.vice.com/read/the-games-that-are-toounderground-to-be-indie>. Acesso em 19 maio. 2016.

KOPAS, Merritt. It's a powerful thing to have people tell me how this games is important to them: interview with Merritt Kopas. 2013. Disponivel na internet por http em: <http://brindlebrothers.blogspot.com.br/2013/03/its-powerful-thing-tohave-people-tell.html>. Acesso em 2 maio. 2016

Creating Space within Games - an interview with Merritt Kopas. 2014. Disponivel na internet por http em: <http://watchtheswitch.tumblr.com/post/ $89981035095 /$ creating-space-within-games-an-interview-with>. Acesso em 2 maio. 2016

Videogames for humans. Estados Unidos: Instarbooks, 2015.

Disponível na internet por http em: <http://forestambassador.com/about>. Acesso em 20 maio. 2015.

OAKES, Kaya. Slanted and enchanted: the evolution of the indie culture. Nova York: Holt paperbacks. 2009.

PAES, Maria Helena. A década de 60: rebeldia, contestação e repressão política. São Paulo: Ática. 2004.

PEDERCINI, Paolo. Toward independence - indiecade 2012. 2012. Disponível na internet por http em: http://www.molleindustria.org/blog/toward-independenceindiecade-2012-microtalk/. Acesso em 13 maio. 2016 
PURDY, Sean. O outro sonho americano. ed.54. São Paulo: História Viva, 2008. Disponível na internet por http em: < http://www2.uol.com.br/historiaviva/ reportagens/o_outro_sonho_americano_imprimir.html>. Acesso em 5 abr. 2016 ROSZAK, Theodore. A Contracultura. Petrópolis: Vozes, 1972.

SILVERMAN, Jacob. Free radical. 2011. Disponível na internet por http em: $<$ http://www.tabletmag.com/jewish-arts-and-culture/81083/free-radical-2>. Acesso em 20 maio. 2016

SOUSA, Getúlio de. Herança da contracultura: a comunidade hippie de Arembepe, Camaçari-Bahia (1970-2012). In: ASSOCIAÇÃO NACIONAL DE HISTÓRIA - ANPUH. 2013, Natal. Anais do XXVII Simpósio Nacional de História. Natal: Anpuh, 2013.

SPENCER, Amy. DIY: The Rise of lo-Fi culture. Estados Unidos: Marion Boyars Publishers, 2008.

WEHR, Kevin. DIY: The Search for Control and Self-Reliance in the 21st Century (Framing 21st Century Social Issues). Nova York: Routledge, 2012. 